\title{
LES RELATIONS DE TRAVAIL DANS LES ENTREPRISES ASSOCIATIVES. SALARIÉS ET EMPLOYEURS BÉNÉVOLES FACE À L'AMBIVALENCE DE LEURS RÔLES
}

\author{
Simon Cottin-Marx
}

I.R.E.S. | « La Revue de l'Ires »

2020/2 n 101-102 | pages 29 à 48

ISSN $1145-1378$

DOI 10.3917/rdli.101.0029

Article disponible en ligne à l'adresse :

https://www.cairn.info/revue-de-l-ires-2020-2-page-29.htm

Distribution électronique Cairn.info pour I.R.E.S..

(C) I.R.E.S.. Tous droits réservés pour tous pays.

La reproduction ou représentation de cet article, notamment par photocopie, n'est autorisée que dans les limites des conditions générales d'utilisation du site ou, le cas échéant, des conditions générales de la licence souscrite par votre établissement. Toute autre reproduction ou représentation, en tout ou partie, sous quelque forme et de quelque manière que ce soit, est interdite sauf accord préalable et écrit de l'éditeur, en dehors des cas prévus par la législation en vigueur en France. Il est précisé que son stockage dans une base de données est également interdit. 


\title{
Les relations de travail dans les entreprises associatives. Salariés et employeurs bénévoles face à l'ambivalence de leurs rôles
}

\author{
Simon COTTIN-MARX ${ }^{1}$
}

Le monde associatif est-il un monde du travail comme les autres ? Pour répondre à cette question, les sociologues ont forgé la notion d'entreprise associative, soulignant que ces organisations employeuses oscillent entre deux registres, celui de l'engagement et celui du monde professionnel. Dans cet article, à partir d'une enquête auprès de salariés et d'employeurs associatifs, l'auteur regarde tour à tour ce que l'engagement fait aux salariés, et ce que le salariat fait à l'engagement des employeurs bénévoles. Car travailler dans le monde associatif, c'est (souvent) travailler " pour la cause » et " avec » des bénévoles. C'est aussi travailler "pour » des bénévoles, pour qui le rôle d'employeur est une responsabilité souvent inattendue et difficile à prendre en charge. Autant de spécificités qui marquent les relations de travail dans les petites entreprises associatives et déstabilisent aussi bien la posture salariale que la posture de l'employeur.

Avec leurs 1,8 million de salariés, les associations sont devenues un employeur de poids dans l'économie. Mais est-ce un employeur comme les autres?

Pour répondre à cette question, les sociologues du travail associatif ont commencé par souligner la spécificité des associations employeuses, qui sont à la fois associations et entreprises. Régies par la loi 1901, elles poursuivent la réalisation d'un projet collectif, ont une gestion désintéressée et mobilisent des bénévoles. Mais en même temps, employeuses, elles ont les

1. Sociologue, post-doctorant au Laboratoire Techniques, Territoires et Sociétés (LATTS). Cet article est le fruit d'un travail de postdoctorat, réalisé avec le soutien de l'Institut national pour la jeunesse et l'éducation populaire (INJEP) et de l'Institut français du monde associatif (IFMA). 
contraintes et les obligations des entreprises classiques. Pour nommer ces structures qui juxtaposent « deux dispositifs distincts et autonomes, celui d'une association et celui d'une entreprise » (Marchal, 1992), les sociologues ont forgé la notion, à partir de ces deux termes qui semblent antinomiques, « d'entreprise associative ». Ainsi, Hély définit « l'entreprise associative [comme] un groupement fondé sur un double contrat : le contrat d'association formalisé par la loi de 1901 et le contrat de travail la rattachant aux règles du Code du travail ». Pour lui, les entreprises associatives sont prises dans une tension, qui les amène à osciller « entre une rationalité en valeur où le projet éthique importe plus que l'ajustement des moyens matériels et une logique instrumentale où le seul calcul rationnel guide l'action » (Hély, 2004).

En conséquence de cette tension, les sociologues repèrent plusieurs spécificités du travail associatif (Hély, Simonet, 2013). Ils soulignent notamment la position ambivalente des salariés, entre engagement et salariat. Simonet-Cusset (2004) propose ainsi de regarder les bénévoles comme des travailleurs et les travailleurs comme des bénévoles. Dans cet article, nous souhaitons approfondir ce cadre d'analyse fécond. Il s'agit pour nous d'envisager les bénévoles, qui siègent dans les instances de direction des entreprises associatives, non seulement comme des travailleurs, mais aussi comme des employeurs. Car, c'est une des spécificités des entreprises associatives, les «patrons » des salariés sont les présidentes et les présidents, et plus largement les membres (forcément) bénévoles des conseils d'administration. Or, être employeur, même bénévole, c'est avoir la charge de la « fonction employeur».

Dans cet article, nous proposons d'étudier les conséquences de cette tension entre logique associative et logique de l'entreprise qui cohabitent dans ces structures sur la relation de travail. Quelles conséquences cette tension a sur la position des acteurs du monde du travail associatif ? Sur la manière dont salariés et «patrons » abordent leurs rôles et fonctions ? Que fait l'engagement au travailleur associatif, et que fait le salariat à l'engagement des responsables bénévoles?

Dans la première partie de cet article, nous présentons une revue de la littérature des principales analyses sur les spécificités du travail dans les entreprises associatives. Dans la seconde partie, nous explorons un angle mort de ces travaux : le rôle des employeurs bénévoles dans les entreprises associatives. Dans cette partie, nous exploitons notre travail d'enquête sur les employés et employeurs de petites entreprises associatives $(<50$ salariés). Nous proposons ainsi de regarder le monde associatif de manière transversale, quel que soit le secteur, mais en focalisant notre regard sur les associations ayant un petit collectif de salariés et où nous postulons que les bénévoles jouent un rôle dans la gestion de la structure. 


\section{Encadré 1}

\section{Méthode}

Nous avons fait le choix de nous intéresser aux entreprises associatives, de moins de 50 salariés, qui regroupent en France $36 \%$ de l'emploi associatif (Tchernonog, Prouteau, 2019), soit environ un peu plus de 600000 salariés.

\section{Entretiens}

Entre 2019 et 2020, 30 entretiens ont été réalisés avec des salariés (la plupart des salariés rencontrés étaient âgés d'une trentaine d'années, mais les interviewés ont entre 26 et 49 ans) et une quinzaine avec des employeurs associatifs, pour la plupart membres d'un syndicat employeur. Ces personnes appartiennent principalement au secteur de la défense des droits et des causes (30\%), à l'humanitaire, social, santé (24\%), au secteur des loisirs (19\%), à celui de l'éducation, de la formation et de l'insertion (14\%), à la culture (8\%). Par ailleurs, les personnes interrogées travaillent ou dirigent des structures de moins de 5 salariés (40\%), entre 5 et 10 salariés (8\%), entre 10 et $20(32 \%)$, et entre 20 et $50(20 \%)$. Une seule structure (dans laquelle deux salariés ont été interrogés) avait plus de 50 salariés, mais ceuxci étaient répartis dans des établissements de petite taille $(<10)$.

Nous avons par ailleurs réalisé six entretiens avec des syndicalistes (Solidaires, CGT, FO, CFDT).

\section{Questions posées lors des entretiens}

Les personnes interrogées étaient questionnées sur leur parcours, sur l'association (et son projet) dans laquelle ils ont travaillé, ainsi que sur les fonctions et métiers occupés, et les activités réalisées dans ce cadre. Ensuite, elles étaient invitées à évoquer leurs conditions de travail, le rapport à la hiérarchie, à l'équipe (dont les bénévoles), et les éventuels conflits du travail. Pour préserver l'anonymat des personnes et des associations, nous avons modifié les noms des personnes et des structures, sauf lorsqu'elles ont donné leur accord pour lever l'anonymat.

\section{La position ambivalente des salariés associatifs : entre salariat et engagement(s)}

Depuis quelques années, les sociologues du travail associatif pointent une particularité du monde associatif : dans ce marché du travail, c'est le salariat « atypique » qui est typique (Hély, 2008a). Comparés au secteur privé et public, les salariés du monde associatif sont fortement touchés par la précarité (Cottin-Marx, 2011), c'est-à-dire par la discontinuité associée à la carence du revenu, ou à la carence des protections. Les sociologues Hély (2009) et Prouteau (2015) y constatent des conditions de travail dégradées avec un recours important aux CDD, aux horaires atypiques, au temps partiel, etc. Mais alors pourquoi ces emplois trouvent-ils preneurs? 


\section{I.1. Travailler " pour la cause »}

Pour répondre à cette question, Preston avance la théorie du « don du travail ». Les salariés seraient « intrinsèquement motivés » (Preston, 1989 ; Narcy, 2009) par le fait de travailler pour des structures œuvrant pour l'« intérêt général ». Pour la chercheuse, les conditions de travail moins bonnes du monde associatif par rapport au secteur privé sont compensées par les bénéfices sociaux produits par les organisations dans lesquels les salariés travaillent. Si les travailleurs sont disposés à accepter un salaire réduit, c'est qu'ils trouvent une compensation dans le fait que leur activité soit source d'« externalités positives ». Autrement dit : servir un projet à but non lucratif apporte une satisfaction morale au travailleur qui justifierait une rémunération plus faible que ce à quoi il pourrait prétendre dans une organisation à but lucratif.

La contribution des individus au bien commun serait une compensation en rétribution symbolique qui rendrait acceptable une rémunération plus faible. Cette idée souligne que le salaire ne constitue pas le critère principal de la reconnaissance professionnelle et que d'autres éléments, comme la satisfaction morale d'accomplir un projet d'utilité sociale, viennent compenser une rémunération plus faible. Les salariés « adhèrent » à la cause défendue par l'association (Dussuet et al., 2007), feraient don de travail en échange d'un emploi pourvoyeurs des externalités positives qui leur permet « de vivre de et pour la cause » (Herman, 2013 ; Cardoso, 2019).

Il est important de noter que, si travailler dans une association offre des rétributions symboliques, qui viennent « compléter » la rémunération pécuniaire, celles-ci n'ont pas la même valeur pour l'ensemble des salariés de la structure. Dans leur travail sur la Croix-Rouge française, Combes-Joret et Lethielleux (2012) montrent que dans cette organisation, les salaires sont plus faibles que dans d'autres structures du secteur privé, mais que « malgré cet écart de rémunération, le choix de rester dans la structure était consenti par certains salariés, voire clairement revendiqué (par deux comptables et une infirmière) comme témoin d'engagement altruiste et comme gage d'un "supplément d'âme". » Cependant, pour certains, le différentiel de salaire apparaît comme une "fatalité ». Ainsi, le rôle des rétributions symboliques ne doit pas faire oublier que l'acceptation d'un emploi est aussi liée à des contraintes économiques ou familiales : qu'il peut relever d'un « faux choix professionnel » (Le Roy et al., 2019). Cela peut aussi être lié à la structuration du marché de l'emploi : le choix d'un emploi plutôt qu'un autre pouvant être contraint par le manque d'offre ou l'employabilité de la personne (Hély, 2008b).

Cet exemple rejoint l'analyse des économistes Le Roy et alii (2019) pour qui « la théorie du don de travail repose sur l'hypothèse centrale d'un choix 
conscient du type d'employeur. Or, si cette idée semble acceptable pour les travailleurs qualifiés n'ayant pas de difficulté d'insertion professionnelle, elle semble assez difficilement défendable pour des salariées non qualifiées des services à la personne par exemple. » L'analyse des données Conditions de travail 2013 de la Dares (Devetter et al., 2017:298) va dans le même sens et fait apparaitre que, « en ce qui concerne les salariés peu qualifiés et contrairement aux cadres, le statut de l'employeur n'est en rien déterminant dans leurs choix originels [d'y travailler]. » Cependant, Devetter et ses coauteurs constatent qu'au cours de l'activité professionnelle, l'importance de la « fierté de travailler» dans des structures associatives grandit pour ces salariés peu qualifiés.

\section{I.2. Travailler " avec » des bénévoles}

«Travailler pour la cause » n'est pas l'apanage des salariés dans les entreprises associatives. Ce serait même plutôt celui des bénévoles, car ces derniers sont les « premiers » dans les associations. Ils le sont historiquement, car les associations ont d'abord été les outils du mouvement social, de la société civile, fondée sur le bénévolat et le militantisme (Cottin-Marx, 2019).

Pour Le Roy et alii (2019), « la figure du travail salarié a été tardive et les salariés ont longtemps été considérés comme des bénévoles, puis des bénévoles indemnisés, motivés avant tout par le projet de l'association et le service aux usagers, et moins par les revenus issus de ce travail » (p. 66). Pour illustrer leur propos, ces auteures mobilisent l'exemple de l'aide à domicile. Elles rappellent que jusque dans les années 1970, le modèle domestique et bénévole prévalait. La salarisation de ce secteur va surtout s'opérer à partir des années 1980, durant lesquelles les associations sont mobilisées dans la lutte contre le chômage et se mobilisent pour répondre aux besoins sociaux croissants.

Si le bénévolat est souvent "premier » historiquement, il l'est aussi dans les projets. À l'origine d'une association, deux personnes bénévoles, au moins, sont nécessaires pour déposer les statuts et définir l'objet de l'association. La loi 1901 prévoit ainsi la présence de bénévoles, même si l'esprit de la loi est parfois détourné, comme dans les associations « unipersonnelles » (Hély, 2009) où les bénévoles sont absents ou "fantômes », ou dans les associations « gestionnaires » où ils ne sont que de pure forme. Comme l'écrivent Dussuet et Flahault (2010), « dans la plupart des associations, le travail bénévole est premier. L'embauche de salariés n’intervient en effet que dans un second temps et d'une façon qui semble induite par les circonstances. »

Ainsi, il convient de ne pas oublier une importante spécificité des entreprises associatives : cela implique souvent de collaborer avec des travailleurs 
bénévoles (Simonet, 2010). Et la frontière est parfois floue entre employeurs travaillant gratuitement, bénévoles et salariés du monde associatif (Hély, Simonet, 2008). Cette " confusion » est très présente entre salariés et bénévoles (Dussuet, Flahault, 2010) en raison des passages de l'un à l'autre, du continuum biographique entre bénévolat et salariat (Dussuet et al., 2007), de la similarité de leurs tâches et de leurs missions.

Cette spécificité entraine une banalisation des heures supplémentaires et une injonction à s'engager sans compter son temps. Au nom de l'« esprit associatif », une part de travail bénévole « va de soi » et est irrémédiablement et implicitement prescrite. Cette non-valorisation du travail par une rémunération pourtant due fait penser à une «bénévolisation du travail » (Simonet, 2010:217). Qui plus est, la proximité entre bénévoles et salariés et la substituabilité du travail salarié au travail bénévole constituent souvent un risque de dénégation de la qualification : si les bénévoles mettent en œuvre gratuitement les mêmes compétences que les salariés, une rémunération élevée de ces derniers semble inutile, voire injuste.

Cette proximité s'illustre particulièrement dans certains processus amenant à l'embauche du salarié. Parfois, les " travailleurs » d'une association sont d'abord les bénévoles et ce n'est que dans un second temps qu'intervient l'embauche, quand les bénévoles ne peuvent plus répondre à la charge de travail. Les postes salariés sont destinés à remplacer ou seconder les bénévoles, ce qui peut facilement entraîner une confusion par la suite entre statut de salarié et bénévole. En effet, cela semble signifier que «n'importe qui », avec "un peu de bonne volonté », peut effectuer le travail. Dans leur étude sur le Secours populaire, Ughetto et Combes (2010) décrivent les associations comme des lieux de travail où les tâches réalisées par les bénévoles ne se distinguent pas nettement de celles réalisées par les salariés. Les salariés associatifs, travaillant avec des bénévoles non rémunérés, ont ainsi des difficultés à obtenir une reconnaissance et même une légitimité professionnelle (Hély, 2009).

\section{I.3. La posture salariale déstabilisée par le registre d'engagement : l'exemple de la syndicalisation}

Entre engagements (le leur, celui des travailleurs bénévoles) et salariat, la frontière est parfois floue et ces salariés-militants peinent parfois à se reconnaitre (et à être reconnus) comme pleinement salariés, ayant des obligations, mais aussi des droits. L'analyse de la difficile syndicalisation dans les petites entreprises associatives est révélatrice de ce phénomène.

Car le syndicalisme dans les petites entreprises associatives ne va pas de soi. Les obstacles à la syndicalisation des salariés sont connus : flexibilité de l'emploi (Wolff, 2008) et recours à la sous-traitance, carrières professionnelles discontinues, importance du chômage, intensification du travail, 
nouvelles formes d'organisation du travail et de management de plus en plus individualisées, etc. sont autant d'obstacles à l'engagement syndical qui concernent l'ensemble du monde du travail (Pernot, 2010 ; Giraud et al., 2018). Mais dans les associations, outre leur présence dans des secteurs économiques difficiles, où les conditions de travail sont souvent dégradées (Devetter et al., 2017), la dimension « engagée » des associations peut également être pointée comme un obstacle à la prise de conscience salariale.

Comme l'explique Nayla A., qui a participé à la création du syndicat Asso en 2009, " pour beaucoup, travailler dans une association c'est avant tout un choix militant, moi la première : je ne veux pas aller bosser chez Total effectivement parce que je ne considère pas que Total agit en faveur de l'intérêt général. Pour autant je ne considère pas que le militantisme doit être un sacrifice et il ne doit pas être un prétexte des employeurs pour se soustraire à leurs obligations. » Pour Nayla A. et sa camarade Elsa M. : « La réalité est là, et c'est la difficulté avec le secteur associatif, comme c'est non lucratif les gens ont l'impression que l'exploitation n'existe pas, mais elle existe. (...) On entend des discours du type : "Que va devenir le projet de l'association? Je tiens au projet de l'association, et si je parle je risque de mettre à mal un beau projet." C'est un discours que l'on entend beaucoup chez les salariés isolés, ils portent le poids de la survie de l'organisation. Il est là le syndrome [de Stockholm], il est vraiment réel. Des gens en souffrance, qui pourraient faire des procès pour harcèlement et qui refusent de le faire car ce serait une trahison » (Cottin-Marx, 2015).

L'enquête que nous avons menée nous amène à rejoindre l'analyse des syndicalistes d'Asso, mais aussi celle du Snaclep-CFDT ${ }^{2}$ (encadré 2) qui avait posé un constat équivalent dans les années 1970. Les salariés associatifs, que nous avons rencontrés et qui sont évoqués par les syndicalistes, ne travaillent pas « par hasard» dans une association. Si, comme nous l'avons vu au début de cette partie, nous ne pouvons pas généraliser ce constat à l'ensemble des salariés associatifs, il y a chez nombre d'entre eux une part d'engagement dans leur travail, qui les amène à faire « don » de travail. Pour le syndicat Asso, c'est dans ce double registre engagement/salariat que réside ce qu'il nomme "syndrome de Stockholm ». Les salariés associatifs soutiennent «la cause ", s'engagent dans l'association, qui pourtant peut leur donner des conditions de travail dégradées. Dans cette perspective qui est souvent la leur, faire valoir ses droits, demander une augmentation, se syndiquer, quelque part " c'est être contre l'association ", c'est poser des difficultés à la réalisation de ses missions, mettre des limites à son engagement, ne plus faire passer «la cause avant tout».

2. Syndicat national du personnel des associations socioculturelles, de loisirs et d'éducation populaire, affilié à la CFDT. 
Dans son travail sur le secteur de l'animation, Mignon (1998) décrit comment ce secteur s'est professionnalisé, est passé d'un monde du travail militant à un monde du travail plus «classique ». Pour ce chercheur, la bascule s'est faite progressivement. Mais il voit, dans le conflit qui a opposé en 1966 le directeur d'un foyer de jeunes travailleuses, propriété d'une congrégation religieuse à Nancy, à son directeur, un moment significatif. Cela tient au fait que le jeune syndicat Snaclep a fait de ce licenciement sans préavis un combat exemplaire. Les syndicalistes «se [sont rendus] à Nancy pour rappeler aux religieuses l'existence du droit du travail. [Qu'il est] interdit de disposer du personnel en vertu de considérations à l'aune de leur propre morale. Le droit au travail concerne tous les salariés, y compris ceux du milieu associatif, et, donc, tous les employeurs, fussent-ils présidents d'association, bénévoles par définition ou clercs»(Mignon, 2006). Les religieuses refusant de respecter les règles du Code du travail, " dans un geste quelque peu iconoclaste, écrit Mignon, le Snaclep [a attaqué], alors, la congrégation aux prud'hommes ». Le chercheur décrit cet évènement comme une «transgression fondatrice ». Le conflit n'ayant pas été réglé à l'amiable, entre personnes recherchant un même idéal, il « [a entraîné] une prise de conscience sur le nécessaire dépassement de la perception des salariés en termes d"“apôtres ou de militants" ». Pour Mignon, jusqu'ici le monde associatif était prisonnier de sa tradition militante, volontaire. Les « permanents » étaient alors toujours considérés comme entre le volontariat et le salariat. Le Snaclep, en rappelant ainsi qu'il s'agit de travail, dépendant du droit du travail, participe à le positionner clairement dans le salariat.

Mignon qualifie ce conflit de «fondateur », du moins pour le champ professionnel de l'animation, cependant nous constatons que la position ambivalente de certains salariés, entre bénévolat et salariat, est toujours d'actualité. L'adhésion des salariés au projet de leur structure employeuse déstabilise leur posture salariale. L'inverse est aussi vrai, la posture salariale et son développement syndical déstabilisent le registre de l'engagement propre aux salariés associatifs. Se syndiquer, défendre ses droits, pour les salariés associatifs, c'est quelque part mettre un frein à l'engagement, déstabiliser l'adhésion au projet de la structure.

\section{II. « Patrons » ou bénévoles ? Les employeurs associatifs face à la fonction employeur}

Dans la partie précédente, nous nous sommes intéressés aux salariés des entreprises associatives, sur lesquels existe une abondante littérature scientifique. Dans cette partie, nous nous appuierons sur l'enquête que nous avons réalisée dans des petites et très petites entreprises associatives, pour aborder cette fois le rôle des employeurs bénévoles. 


\section{Texte du Snaclep-CFDT, 1972}

«Quand on travaille dans l'éducation populaire (...), on se ressent comme un travailleur-pas-comme-les-autres : pourquoi ? (...) Progressivement, on assimile les objectifs et l'idéologie de l'association employeur. On sent que ça ne va pas bien dans l'éducation populaire, que le gouvernement ne donne pas assez de subventions, on n'est pas d'accord avec la politique du gouvernement en matière de jeunesse, mais comment lutter ? Alors on accepte des salaires bas, des conditions de travail difficiles parce qu'on est dans le "social", parce qu'on pense que, là où on travaille, on peut œuvrer pour une société plus juste et qu'il faut se sacrifier un peu.

En même temps, regrettent les animateurs, on s'interdit de trop bouger parce qu'on risque de perdre sa place, de détruire son propre instrument de travail alors que celui-ci permet de satisfaire son aspiration personnelle à construire un bout de société qui échapperait aux contradictions de la société en général (...). Les patrons n'apparaissent pas comme des patrons (...). La direction ? Mais ce sont des employés, embauchés et rétribués par un conseil d'administration, ce sont des cadres qui ont délégation de pouvoir du conseil d'administration. Le conseil d'administration ? Mais ce sont des bénévoles, "théoriquement" élus par une assemblée générale. En fait, dans la pratique, on sait bien que ce sont des notables (médecins, avocats, banquiers, membres du clergé) (...) qui servent de caution et de carte de visite auprès des pouvoirs publics (...). Voilà ce qui est vécu et ce qui se dit dans les associations lorsqu'on parle d'organisation et de lutte des travailleurs de ce secteur. Le rapport employeur-employé est d'autant plus difficile à dévoiler qu'on a l'illusion, de part et d'autre, de participer à la transformation des rapports de travail traditionnels et, par-là, de "faire avancer les choses". "

Source : Mignon (1998:215).

Nous verrons tout d'abord que, dans les entreprises associatives, la fonction employeur peut être occupée d'une multitude de manières, collectivement ou individuellement, par des bénévoles ou des salariés, même si in fine les dirigeants bénévoles sont responsables de la structure (II.1). Ensuite, nous verrons que le rôle d'employeur est une responsabilité inattendue (II.2), difficile à prendre en charge (II.3) et que cela amène même certains employeurs bénévoles à être dans le déni de leurs responsabilités (II.4).

\section{II.1. Qui occupe la fonction employeur dans les associations ?}

C’est une des spécificités des entreprises associatives : contrairement aux entreprises privées lucratives, elles ne sont pas dirigées par des détenteurs de capitaux, mais par des bénévoles. Ces derniers composent les instances (bureau, conseil d'administration, assemblée générale, etc.) de 
l'organisation. Le représentant légal de l'association (généralement le président ou la présidente) a le pouvoir d'ester en justice et de signer les contrats au nom de l'association. C'est lui qui a le pouvoir de signer les contrats de travail, qui occupe la « fonction employeur » et exerce le pouvoir patronal (disciplinaire, réglementaire, économique). Et en tant qu'employeur, ses responsabilités « sont multidimensionnelles. Elles peuvent être d'ordre social et sanitaire, budgétaire, financier, salarial» (Le Roy et al., 2019:66). $\mathrm{La}$ « complexité » de cette fonction employeur fait qu'elle est souvent partagée ou déléguée, notamment à certains salariés (directeur ou directrice, DRH, etc.). Ces transferts de responsabilités peuvent être plus ou moins complets, plus ou moins contrôlés suivant la taille, mais aussi le secteur, l'histoire, les parcours des dirigeants bénévoles, etc., de l'association.

Dans la revue Mouvements, Hély et alii (2015) s'interrogent : "Qui est l'employeur? » Qui exerce la fonction employeur dans les entreprises associatives? Les bénévoles ou les salariés ? Les sociologues montrent que le statut du responsable est fortement dépendant de la branche professionnelle de l'entreprise. Dans le sport, ils sont plus de $90 \%$ à être des dirigeants bénévoles, «alors qu'ils sont tous salariés dans le logement social et le tourisme social et familial ».

Ces auteurs montrent également que la taille de l'entreprise est le facteur déterminant de l'organisation du rapport salarial. Dans la continuité de ces résultats, notre travail de thèse (Cottin-Marx, 2016) nous a permis de constater des résultats similaires : en moyenne, dans les entreprises associatives (tous secteurs confondus) au-delà de quatre salariés, le dirigeant effectif de la structure est plus souvent un salarié qu'un bénévole. Une tendance qui se renforce avec le nombre de salariés dans la structure. Cela ne signifie pas que les présidents, et plus largement les bénévoles, ne jouent plus aucun rôle dans les associations de plus de quatre salariés, mais que ce sont plus souvent des salariés que des bénévoles " qui ont réellement en charge la continuité de l'action [et] tendent (...) à devenir les véritables porteurs et défenseurs des valeurs du projet» (Le Roy et al., 2019).

Il faut cependant faire attention à ne pas tirer de conclusions trop strictes, car comme nous l'explique la directrice d'une structure de quatre salariés œuvrant dans le secteur social, « le partage des rôles [entre elle et le conseil d'administration de la structure] n'est pas toujours clair ». La « fonction employeur » n'est pas toujours confiée de manière claire et formelle. Elle est parfois partagée entre bénévoles, avec un ou plusieurs salariés. Il existe une multitude de configurations. La directrice que nous avons citée doit, par exemple, selon les tâches et les périodes, composer avec son conseil d'administration, plus ou moins présent, plus ou moins volontaire. Lors d'un entretien, Robert Baron, qui fut à la tête du syndicat employeur 
de l'animation, le Conseil national des employeurs d'avenir (CNEA ${ }^{3}$ ), auquel adhèrent essentiellement des petites entreprises associatives $(<10)$, et qui est aujourd'hui conseiller prud'homal, constate que dans les petites entreprises associatives « il y a un mélange des genres. Quand il y a un directeur, il est un peu coincé entre le CA et les salariés. Il participe, et écrit même souvent le projet. Il est partie prenante du projet politique. (...) En même temps on lui délègue la gestion des ressources. Et il lui faut une certaine indépendance pour recruter, sanctionner, etc. Mais ce n'est pas toujours respecté, ça crée des conflits. Souvent le mandat qui lui est donné n'est pas très clair. »

Ainsi, dans les petites entreprises associatives, il n'est pas rare qu'un flou existe sur les responsabilités de chacun, que la fonction employeur soit " partagée ", de manière mouvante, entre direction bénévole et salariée. C'est notamment ce que montre Lebon (2016), à travers l'exemple d'un centre social où la direction salariée occupe la fonction employeur. Or, cette situation n'est pas «systématique » : dans des centres sociaux équivalents, elle peut aussi bien être prise en charge par les directeurs ou directrices que par le conseil d'administration (Cortesero, 2013).

Dans la suite de cet article, nous allons nous intéresser aux conséquences d'une direction bénévole sur le travail des salariés des petites entreprises associatives, là où le «flou » sur la fonction employeur est généralement présent.

\section{II.2. Être employeur bénévole, une responsabilité inattendue}

Dans leur « Manifeste », Didier Jacquemain et David Cluzeau, respectivement président et délégué général du syndicat employeur CNEA qui rassemble essentiellement des structures de moins de 10 salariés, écrivent : « Nous n'entrons, le plus souvent, pas en association pour générer une activité économique productive, mais pour répondre à des besoins sociaux. L'intention se veut donc davantage altruiste que lucrative. A fortiori, la question de l'emploi n'est pas envisagée en premier lieu ${ }^{4}$. » Pour le CNEA, les bénévoles associatifs entrent en association pour conduire un projet et non pour être employeur. Une idée que confirme le Syndicat des mouvements et associations (SMA-CFDT), qui défend pour sa part les intérêts des salariés. Pour son secrétaire général, Alain Pellé, « les bénévoles des petites associations n'ont pas envie de s'occuper du personnel. Ce sont des militants du projet, les charges qui leur incombent, ils s'en désintéressent en général » (Russo, 2020:147).

3. Représentatif dans la branche de l'animation, des foyers de jeunes travailleurs, du sport, et du tourisme social et familial.

4. CNEA, Le Manifeste pour un entrepreneuriat d'intérêt général, citoyen et sans frontières, novembre 2018. 
Les présidents et présidentes d'associations découvrent parfois brutalement ce rôle d'« employeur ", comme cette dirigeante bénévole d'une association sportive. Alors qu'elle vient tout juste de prendre la tête de la structure, elle "se retrouve avec un prud'homme ». Elle se dit « démoralisée » par cette situation inattendue. En s'engageant bénévolement dans la structure et en prenant sa présidence, elle n’avait pas pris la mesure des responsabilités qui lui incombaient : être la représentante de l'association, même devant les tribunaux.

Comme l'écrivent Hély et alii (2015:69), « la fonction employeur dans le contexte associatif n'est pas complètement assumée et, parfois même, "douloureuse" à incarner, notamment pour les militants de la première heure, désormais dans l'obligation d'incarner une réelle fonction d'employeur alors qu'ils s'étaient engagés par adhésion au projet associatif.» Ils ajoutent que les employeurs associatifs « sont souvent des militants qui ont choisi ou accepté d'endosser les responsabilités d'employeur pour mieux défendre leur "cause", sans toujours avoir conscience qu'ils pourraient rendre compte de leurs pratiques devant le Conseil de prud'hommes. Ils militaient parfois dans le même syndicat que "leurs" salariés et se trouvent brusquement en situation d'un "patron" qui doit sanctionner, arbitrer et réglementer les pratiques des salariés. Paradoxalement, si être administrateur d'une association est en général un choix, être employeur ne l'est pas souvent. » Patronat, syndicat et sociologues s'accordent sur ce constat : pour les bénévoles employeurs, l'emploi n'est pas la préoccupation initiale à leur engagement. La fonction employeur vient même en creux de leur engagement pour le projet. Ces responsabilités « ne vont pas de soi » (Cardoso, 2019), apparaissent souvent comme inattendues et sont rarement une priorité.

\section{II.3. La difficile prise en charge de la fonction employeur}

Si le premier constat partagé est que la « fonction employeur » est souvent inattendue, un deuxième constat semble faire l'unanimité : les dirigeants bénévoles ont globalement une méconnaissance des obligations de l'employeur.

\section{Une fonction vue comme chronophage et décourageante}

La prise en charge de la fonction employeur par les dirigeants bénévoles rencontre de nombreux obstacles. Le premier est l'absence de compétences et de formation des bénévoles pour remplir cette mission. Même si l'on observe une surreprésentation des catégories sociales moyennes et supérieures chez les présidents d'associations (les cadres moyens et supérieurs représentent $40 \%$ des présidents et les chefs d'entreprise 7,5\%) (Tchernonog, Prouteau, 2019), ils ne sont pas forcément des professionnels des secteurs associatifs dans lesquels ils s'engagent. Et, s'ils méconnaissent souvent les 
obligations relatives à leur statut d'employeur, ils n'ont pas forcément le temps de se former et de remplir pleinement le rôle d'employeur, d'autant plus que celui-ci peut être particulièrement chronophage.

Dans une association environnementale qui se réclame de l'éducation populaire (10-20 salariés), où le conseil d'administration joue un rôle important dans l'organisation du travail malgré la présence d'un directeur, un salarié, délégué du personnel syndiqué à Asso, constate : "Les bénévoles du conseil d'administration ne sont pas formés [à être employeurs]. Ce n'est pas facile de leur dire de se former. (...) Former les bénévoles sur plusieurs jours c'est compliqué. C'est déjà difficile de les mobiliser sur les projets de l'association, alors leur demander de dégager des jours pour se former aux questions salariales et de temps de travail... » Pour ce salarié, demander aux employeurs bénévoles de se former, de donner du temps pour la fonction employeur, c'est prendre le risque de les décourager, qu'ils préfèrent se désengager alors qu'il « est déjà difficile de renouveler le CA de notre structure $»$.

Ainsi, si la défense du projet apparaît comme moteur de l'engagement des dirigeants bénévoles, est pourvoyeuse de rétributions symboliques (Gaxie, 2005), la prise en charge de la fonction employeur se révèle plutôt comme un frein. Le syndicaliste d'Asso en est bien conscient : insister auprès des bénévoles pour qu'ils s'investissent davantage sur la question salariale, c'est prendre le risque de les décourager.

La prise en charge de l'emploi par les dirigeants bénévoles apparait comme lourde et peut aller jusqu'à entraîner une "perte de sens » des bénévoles. Dans leur travail sur les associations féministes, Flahault et Loiseau (2009) montrent comment l'arrivée de salariées dans l'association transforme l'organisation. "Dans certaines associations, on assiste alors à une démission des militantes qui fuient à la fois un climat tendu et une organisation dans laquelle elles ne se retrouvent plus, ni sur le plan des valeurs défendues ni sur celui de la pratique, notamment lorsqu'elles sont confrontées à la nécessité de gérer des salariées sans y avoir été préparées. Certaines regrettent l'affaiblissement, voire la perte, de la dimension conviviale, et considèrent que les fondements de l'association sont battus en brèche. Celles qui restent regrettent parfois d'être accaparées par les tâches de gestion au détriment de la réflexion sur les missions et les pratiques de l'association. En effet, avec le salariat, la recherche de financements - avec notamment la perpétuelle obligation de renouvellement des projets - devient une hantise que partagent dans des proportions variables direction salariée et encadrement militant »(Flahault, Loiseau, 2009).

Être employeur associatif prend du temps, amène à faire des tâches administratives peu motivantes. La position « d'employeur » est aussi parfois mal vécue par les dirigeants bénévoles. C’est ce qu’explique le délégué du 
personnel cité précédemment : «Dans mon association, les entretiens DP [avec le directeur et des membres du bureau - composé de syndicalistes] sont compliqués. Ils nous reprochent d'être trop formels, de mettre les administrateurs en position de "Medef"... Ils vivent ça trop fort, et on doit doser ce que l'on peut dire ou pas. »

\section{Des employeurs qui projettent leur expérience professionnelle sur leurs salariés}

Les dirigeants bénévoles associatifs sont souvent âgés, puisqu'ils étaient $41 \%$ à avoir plus de 65 ans en 2017 (Tchernonog, Prouteau, 2019). Qui plus est, l'âge du président augmente avec le nombre de salariés, ce qui confirme, écrivent Tchernonog et Prouteau, que « le rôle de l'expérience et de la qualification semble toujours aussi prégnant à la tête des grandes associations ». Ces derniers ont donc la plupart du temps une expérience professionnelle. Or, cela n'est pas neutre sur leur manière d'occuper la fonction employeur. Ainsi, pour David Cluzeau, délégué général du syndicat employeur CNEA, " quand on entre en association, on entre pour conduire un projet, pas pour être employeur. Donc l'emploi n'est pas la préoccupation initiale. C'est pourquoi les employeurs associatifs peuvent être de mauvais employeurs. Et ceux qui ont la fibre employeur ne sont pas forcément meilleurs, car ils ont tendance à transposer leur monde professionnel. » Notre enquête nous amène à rejoindre cette analyse : les employeurs bénévoles ont tendance à projeter leur expérience salariale ou patronale sur les associations qu'ils dirigent.

Cette « transposition du monde professionnel » des dirigeants bénévoles peut être aussi illustrée à travers l'exemple d'une association de défense du milieu agricole. Pour un salarié de cette structure : «Ce qui est particulier par rapport à nos employeurs [bénévoles], c'est que c'est des agriculteurs. Certains n'ont jamais été salariés, d'autres sont employeurs. Ils ont souvent un rapport lointain au salariat, encore plus lointain quand il s'agit d'occuper la fonction employeur. Ils nous disent souvent: "Nous on est bénévoles, on prend du temps sur nos fermes, si c'est relou on ne viendra plus s'occuper de vous". (...) Après, il y a aussi la question du temps de travail. Les agriculteurs travaillent 70 heures par semaine, et nous, quand on demande à compter nos heures, ça a l'air mesquin. » Ce cas est intéressant, car la projection de l'expérience professionnelle ne se fait pas forcément directement ni n'est toujours explicitée. Dans ce cas, c'est le salarié qui prend en compte l'expérience professionnelle de ses employeurs bénévoles.

\section{Des employeurs qui projettent leur engagement sur l'organisation du travail}

Les dirigeants bénévoles ont tendance à projeter leur expérience professionnelle, ils projettent aussi leurs pratiques d'engagement. Face aux salariés, il n'est pas rare qu'ils rappellent qu'ils consacrent du temps et de 
l'énergie gratuitement (Ughetto, Combes, 2010), ce qui d'ailleurs, comme nous l'avons vu précédemment, peut peser sur les salariés comme une « injonction à s'engager sans compter son temps » (Hély, 2009).

Lors de notre enquête, nous avons rencontré la salariée d'une association humanitaire, dont le directeur était aussi créateur de l'entreprise associative. Enthousiaste lors de sa prise de poste, elle a vite déchanté : «Le respect du droit du travail, c'était toujours remis en question. Comme si l'accès aux droits était une faveur. (...) Par exemple, j’ai commencé à travailler plus d'un mois avant le début de mon contrat. Mais trois mois après, quand j'ai demandé si je pouvais avoir des congés entre Noël et le jour de l'An, il y a eu un grand silence, comme si je demandais quelque chose d'extraordinaire. Et je me suis sentie obligée de me justifier, que j'avais fait un an sans vacances. Il m'a répondu: "ma pauvre", sous-entendu que lui faisait des sacrifices depuis plusieurs années pour monter son association et que je devais faire pareil. (...) Petit à petit, je me suis mise à travailler comme eux : 9 heures-20 heures. (...) Le vendredi soir, on faisait des réunions, et ils demandaient une note pour le lundi. Je faisais du mimétisme, pour m'intégrer dans cette boîte où l'on travaillait comme des malades. (...) De la part des employeurs, il y avait une forme de culpabilisation. Ils disaient : "Nous, on ne compte pas nos heures, car le projet nous plait, on sauve le monde." Et ils faisaient sentir aussi que si ce n'était pas moi qui travaillais ce week-end, ils n'allaient pas s'en sortir, qu'ils étaient déjà submergés, et que lui il travaille déjà tout le temps et ne va pas tenir. Il y avait des regards lourds si les gens partaient à 18 heures. » Les dirigeants de cette association, qui font d'importants « dons » de travail, attendent de leurs salariés un « engagement » équivalent (encadré 3).

\section{II.4. Des employeurs dans le déni de leurs responsabilités}

Les responsabilités découlant de la fonction employeur sont souvent inattendues pour les dirigeants bénévoles. Sa prise en charge s'avère aussi difficile. Pour Alain Pellé, du syndicat SMA-CFDT, certains employeurs refusent même cette fonction, et sont dans le " déni de la situation d'employeur. " Amer, lors d'un entretien il ajoute : «Ils savent très bien s'organiser en collectif pour faire pression pour faire des campagnes de communication. Par contre, quand il s'agit d'assumer la fonction employeur et de se penser dans les termes d'une chambre patronale, alors là, il n'y a plus personne. »

Lors de notre enquête, nous avons constaté plusieurs exemples de « déni», certains employeurs ne s'assumant pas comme tels. Dans ces petites structures employeuses, où les régulations sont souvent affinitaires et informelles, certains employeurs sont mal à l'aise dans la relation de subordination avec leurs salariés, peuvent faire comme si les hiérarchies salariales n'existaient pas. Qui plus est, dans ces structures parfois «militantes », la séparation entre 


\section{Salarié ou militant ? \\ La double casquette des membres d'une association d'éducation populaire}

Pierre est employé depuis plus de cinq ans dans une association d'éducation populaire dont l'une des principales tâches est de faire de la formation. Sa structure est composée d'une quarantaine de salariés et d'une dizaine de personnes ayant des statuts variés (volontaires, stagiaires, intervenants en contrat d'engagement éducatif). II occupe pour sa part un poste technique à plein temps, est syndiqué et délégué du personnel. II constate que sa structure est victime d'un gros turn over.

« II y a des gens qui partent très vite. Car c'est un rythme assez soutenu. On a une direction qui travaille beaucoup trop. Le directeur et la directrice adjointe, c'est des gens qui travaillent facilement 70 heures par semaine... et du coup, l'attente, c'est qu'on travaille beaucoup. Ce n'est pas formalisé directement, explicitement. Mais l'attente est là. Et on est quelques personnes à vouloir respecter nos heures. (...) Mais la plupart des personnes qui arrivent se font absorber et passent leur temps à travailler. Et du coup c'est un attendu. Les gens qui n'ont pas envie de ça se retrouvent à partir... et en plus, [il y a une] pression financière globale et une tendance à individualiser cette pressionlà. (...) Quand les gens partent, la direction parle de "personnes fragiles", elle psychologise les choses (...), alors qu'en réalité ces personnes étaient confrontées à des postes de travail intenables, très difficiles. »

« On est dans une gestion hyper à l'affectif. Sur deux niveaux. Les problèmes ne sont jamais nommés comme institutionnels, mais comme des problèmes de personnes. Et quand on renvoie ces choses à la direction, ils renvoient sur la dimension affective : "Je fais ce que je peux". Alors qu'on ne lui parle pas en tant que personne, mais en tant que directeur... "Je travaille déjà beaucoup". Mais ce n'est pas le débat. Cette question de l'affectif est forte et je pense que c'est lié, car dans une structure militante, les gens sont attachés à la structure, c'est important ce que l'on fait, c'est utile... il y a tous les mécanismes de justification. Et ça marcherait moins si les gens n'étaient pas attachés à la structure. (...) Beaucoup de collègues (sauf les postes techniques et administratifs) sont des anciens bénévoles, des personnes qui sont devenues salariées, qui font des allers-retours entre les deux. Avec un rapport toujours ambigu à ça. (...) Actuellement, dans mes collègues, il y a l'ancien président de la structure, une personne qui est en couple avec un membre du conseil d'administration (CA). II y a aussi deux anciens membres du CA. Ils sont nombreux à changer de casquette. » 
bénévoles et salariés n'est pas toujours nette. Certaines personnes passent d'un statut à l'autre, d'employeur à salarié, de salarié à bénévole. Dans ces organisations de personnes, la proximité est parfois grande, comme le raconte ce salarié associatif : "J'allais manifester le samedi avec lui, et je le revoyais le lundi comme employeur. » Entre " camarades », c'est parfois difficile de s'assumer comme « patron ». C'est le cas par exemple, pour ce syndicaliste, président du conseil d'administration d'un comité d'entreprise, pour qui se voir coller l'étiquette d'« employeur » est une aberration.

Ainsi, travailler « pour» des bénévoles n'est pas neutre. C’est faire face à des employeurs qui ne se reconnaissent pas toujours comme tels, pour qui la fonction employeur est souvent inattendue et pour qui sa prise en charge est difficile.

\section{Conclusion}

Regarder tour à tour ce que l'engagement fait aux salariés, et ce que le salariat fait à l'engagement des employeurs bénévoles nous permet d'aborder la complexité des relations de travail dans les entreprises associatives. De constater que la posture du salarié et la posture d'employeur sont déstabilisées par l'ambivalence de leurs rôles. Ils oscillent entre deux registres. Le premier est celui de l'engagement, lié au monde associatif. Le second est " professionnel », relatif à l'entreprise. Pour filer la métaphore théâtrale, nous pourrions dire qu'ils occupent des rôles de composition, qui ne sont pas toujours simples à occuper.

Ce double registre laisse son empreinte sur les relations de travail dans les petites entreprises associatives. Il participe à escamoter les rapports de domination inhérents à la relation salariale (Hély et al., 2015) et ce d'autant plus que les rapports sont souvent affinitaires. Est-ce pour autant qu'il n'y a pas de conflits du travail dans les associations? Statut n'est pas vertu : le régime de l'entreprise «ne garantit rien quant au recul de l'oppression » (Clot, 2019). Cependant, le registre de l'engagement, qui se superpose à celui de l'entreprise, participe à ce qu'ils soient déplacés. Il amène les employeurs à ne pas voir leurs salariés seulement comme salariés, et les salariés à ne pas voir leurs employeurs comme patrons. Ils ont un engagement en commun, ils sont «dans le même bateau » (Mignon, 1998). Lorsque les salariés associatifs et leurs employeurs se voient poser la question « qui est le patron des associations ? (Cottin-Marx et al., 2015), ils désignent moins les membres des instances dirigeantes de l'association que les financeurs, a fortiori les pouvoirs publics qui sont d'importants bailleurs de fonds de leurs entreprises associatives. Le registre de l'engagement participe ainsi à détourner les conflits, à les placer entre associations et pouvoirs publics, qui sont vus in fine comme les véritables employeurs. 


\section{Références bibliographiques}

Cardoso A. (2019), « Quand les patronnes sont bénévoles : conflictualités au Planning Familial ", La Nouvelle Revue du Travail, n 15, https://doi. org/10.4000/nrt.5780.

Clot Y. (2019), " Le travail : un objet politique sans sujet ? », in Supiot A. (dir.), Mondialisation ou globalisation? Les leçons de Simone Weil, Collège de France, Paris, https://www.college-de-france.fr/site/alain-supiot/symposium2017-06-13-10h00.htm.

Combes-Joret M., Lethielleux L. (2012), « Le sens du travail à la Croix-Rouge française : entre engagement pour la cause et engagement dans le travail », Revue internationale de l'économie sociale : Recma, $\mathrm{n}^{\circ} 323, \mathrm{p} .64-81$, https://doi.org/10.7202/1018345ar.

Cortesero R. (2013), « Les centres sociaux, entre participation et cohésion sociale », Dossier d'étude, n¹60, Cnaf, https://bit.ly/3iRwysD.

Cottin-Marx S. (2011), "Précarité et monde du travail associatif », mouvements. info, https://mouvements.info/precarite-et-monde-du-travail-associatif/.

Cottin-Marx S. (2015), "Créer un syndicat pour défendre les salariés du secteur associatif : entretien avec Nayla A. et Elsa M. du syndicat Asso ", Mouvements, $\mathrm{n}^{\circ}$ 81, p. 77-83, https://doi.org/10.3917/mouv.081.0077.

Cottin-Marx S. (2016), Professionnaliser pour " marchandiser " (et inversement), Thèse, Université Paris Est Marne-la-Vallée, https://hal.archives-ouvertes. fr/tel-01416840.

Cottin-Marx S. (2019), Sociologie du monde associatif, Paris, La Découverte.

Cottin-Marx S., Grisoni A., Roueff O. (dir.) (2015), «Qui est le patron des associations ? ", dossier, Mouvements, $\mathrm{n}^{\circ}$ 81, https://www.cairn.info/revuemouvements-2015-1.htm.

Devetter F.-X. (dir.), Abasabanye P., Bailly F., Barrois A., Brolis O., Chapelle K, Dussuet A., Lene A., Nirello L., Puissant E., Prouteau L. (2017), Les salariées du secteur associatif : des conditions de travail et des relations de service spécifiques?, Rapport pour la Dares.

Dussuet A., Flahault É. (2010), « Entre professionnalisation et salarisation, quelle reconnaissance du travail dans le monde associatif ? ", Formation Emploi, $\mathrm{n}^{\circ} 111$, p. 35-50, https://www.cairn.info/revue-formation-emploi2010-3-page-35.htm.

Dussuet A., Flahault E., Loiseau D. (2007), Quelle gestion des ressources humaines dans l'économie sociale ? Entre bénévolat et professionnalisation, la place du travail dans les associations, Rapport final, DIIESES, octobre, https://halshs.archives-ouvertes.fr/halshs-00195111/document.

Flahault E., Loiseau D. (2009), « Que fait le salariat au militantisme dans les associations féministes ? ", Amnis, $n^{\circ} 8$, https://halshs.archives-ouvertes.fr/ halshs-00366471/document.

Gaxie D. (2005), « Rétributions du militantisme et paradoxes de l'action collective », Swiss Political Science Review, vol. 11, n 1, p. 157-188, https://doi. org/10.1002/j.1662-6370.2005.tb00051.x.

Giraud B., Yon K., Béroud S. (2018), Sociologie politique du syndicalisme : introduction à l'analyse sociologique des syndicats, Paris, Armand Colin. 
Hély M. (2004), « Les différentes formes d'entreprises associatives », Sociologies pratiques, p. 27-51, https://halshs.archives-ouvertes.fr/halshs-00009569/ document.

Hély M. (2008a), « À travail égal, salaire inégal : ce que travailler dans le secteur associatif veut dire ", Sociétés contemporaines, n69, p. 125-147, https:// doi.org/10.3917/soco.069.0125.

Hély M. (2008b), « L'économie sociale et solidaire n'existe pas », La Vie des idées, 11 février, https://laviedesidees.fr/L-economie-sociale-et-solidaire-n-existepas.html.

Hély M. (2009), Les métamorphoses du monde associatif, Paris, Puf.

Hély M., Simonet M. (2013), Le travail associatif, Paris, Presses universitaires de Paris Nanterre.

Hély M., Rétif S., Simonet M. (2015), "Figures de l'employeur et formes du "dialogue social" dans les entreprises de l'ESS ", Mouvements, $n^{\circ} 81$, p. 116-125, https://doi.org/10.3917/mouv.081.0116.

Hély M., Simonet M. (dir.) (2008), "Splendeurs et misère du travail associatif », dossier, Les Mondes du travail, $\mathrm{n}^{\circ} 5$.

Herman É. (2013), « Militer en travaillant contre les violences conjugales », Cahiers du genre, $\mathrm{n}^{\circ} 55$, p. 65-87, https://doi.org/10.3917/cdge.055.0065.

Lebon F. (2016), " De la démocratie en centre social : enquête sur les relations professionnelles dans une association de quartier ", Revue internationale de l'économie sociale : Recma, $\mathrm{n}^{\circ} 340$, p. 93-108, https://doi. org/10.7202/1037405ar.

Le Roy A., Puissant E. (dir.), Devetter F.-X., Vatan S. (2019), Économie politique des associations : transformations des organisations de l'économie sociale et solidaire, Louvain-la-Neuve, De Boeck.

Marchal E. (1992), « L'entreprise associative entre calcul économique et désintéressement ", Revue française de sociologie, vol. 33, $\mathrm{n}^{\circ} 3$, p. 365-390, https://doi.org/10.2307/3322268.

Mignon J.-M. (1998), La lente naissance d'une profession : les animateurs de 1944 à 1988, Thèse de doctorat d'histoire contemporaine, Université Michel-deMontaigne Bordeaux 3, http://www.theses.fr/1998BOR30002.

Mignon J.-M. (2006), «Quand les salariés d'associations de tourisme social, de loisirs et d'animation socioculturelle se syndiquèrent à la CFDT, février 1964-décembre 1968 », in Tartakowsky D., Tétard F. (dir.), Syndicats et associations : concurrence ou complémentarité ?, Rennes, Pur, p. 181189, http://books.openedition.org/pur/25333.

Narcy M. (2009), "Les salariés du secteur associatif sont-ils davantage intrinsèquement motivés que ceux du secteur privé ? ", Économie \& Prévision, n 188, p. 81-99, https://doi.org/10.3406/ecop.2009.7901.

Pernot J.-M. (2010), Syndicats : lendemains de crise ?, Paris, Gallimard ( $1^{\text {re }}$ parution 2005).

Preston A.E. (1989), "The Nonprofit Worker in a For-Profit World ", Journal of Labor Economics, vol. 7, n 4, p. 438-463, https://www.jstor.org/ stable/2535137. 
Prouteau L. (2015), « Les emplois occasionnels dans les associations d'économie sociale », Revue internationale de l'économie sociale : Recma, $n^{\circ} 338$, https://bit.ly/34Oik6P.

Russo P.-D. (2020), Souffrance en milieu engagé. Enquête sur des entreprises sociales, Paris, Faubourg Éditions.

Simonet M. (2010), Le travail bénévole : engagement citoyen ou travail gratuit ?, Paris, La Dispute.

Simonet-Cusset M. (2004), «Penser le bénévolat comme travail pour repenser la sociologie du travail », La Revue de I'IRES, n 44, p. 141-155, https://bit. ly/34T4P5T.

Tchernonog V., Prouteau L. (2019), Le paysage associatif français : mesures et évolutions, Paris, Dalloz.

Ughetto P., Combes M.-C. (2010), « Entre les valeurs associatives et la professionnalisation : le travail, un chaînon manquant ? », Socio-Logos, $n^{\circ} 5$, https:// doi.org/10.4000/socio-logos.2462.

Wolff L. (2008), « Le paradoxe du syndicalisme français : un faible nombre d'adhérents, mais des syndicats bien implantés ", Dares, Premières Synthèses, $n^{\circ}$ 16.1, avril, https://travail-emploi.gouv.fr//MG/pdf/2008.04-16.1.pdf. 